\title{
Red Cell Distribution Width as a Severity Marker on the Outcome of Patients with Acute Kidney Injury on Renal Replacement Therapy
}

\author{
Sunil Nanjarapalle ${ }^{1}$, Aloka Samantaray ${ }^{2}$, Sivakumar Vishnubhotla ${ }^{3}$
}

\begin{abstract}
Background: Acute kidney injury (AKI) requiring dialysis is associated with high mortality and morbidity. Red blood cell distribution width (RDW) has been shown as a predictor of mortality in different subsets of patients admitted to intensive care unit (ICU). This study compares the predictive ability of RDW and other severity illness prognostic models on 30 days mortality in adult patients admitted to ICUs with AKI necessitating dialysis.

Materials and methods: Thirty patients were evaluated using five different prognostic scoring models. Sequential organ failure assessment (SOFA) score, acute tubular necrosis-individual severity index (ATN-ISI), version II of acute physiology and chronic health evaluation (APACHE II), vasoactive-inotropic score (VIS), version II of simplified acute physiology score (SAPS II), and RDW as a marker were used to prognosticate the severity of illness. The scores were calculated using the values of clinical and laboratory parameters at the time of admission.

Results: The prognostic abilities of the scores were compared for their discriminatory power using receiver-operating characteristic (ROC) curves. The area under the ROC curve (AROC) of RDW was 0.904 , SOFA score was 0.828 , ATN-ISI was 0.743 , SAPS was 0.857 , and APACHE II score was 0.828. Vasoactive-inotropic score has the lowest discriminatory power with AROC of 0.487 . Red blood cell distribution width has a strong and significant correlation with APACHE II and SOFA scores and a weak relation with ATN-ISI score and SAPS II.

Conclusion: Red blood cell distribution width has a better predictive ability than other disease severity scoring systems to predict mortality in an adult AKI patient admitted to ICU with need for renal replacement therapy (RRT).

Keywords: Acute kidney injury, Disease severity, Hospital mortality.

Indian Journal of Critical Care Medicine (2020): 10.5005/jp-journals-10071-23342
\end{abstract}

\section{INTRODUCTION}

Acute kidney injury (AKI) is not only a reason for intensive care unit (ICU) admission but also a serious complication among ICU patients. Acute kidney injury requiring dialysis has been consistently associated with detrimental impact on patient's outcome in terms of morbidity and mortality. Oxidative stress and inflammation are predominant component involved in the pathogenesis of $\mathrm{AKI}^{1,2}$ Red blood cell distribution width (RDW) is an expression of the variation in size of the red blood cells (RBCs) that make up the total population in an individual patient. Clinical evidences suggest that both inflammation and oxidative stress reduces RBCs survival and suppress their maturation resulting in the release of large premature RBCs into circulation, contributing to elevated RDW. ${ }^{3}$ Estimation of RDW is a relatively inexpensive simple laboratory investigation that can be routinely obtained while sending request for a routine hemogram.

Red blood cell distribution width was shown to be an important prognostic indicator in various conditions, such as in patients with severe sepsis and septic shock. ${ }^{4}$ Both sepsis and septic shock have been implicated as major predictor of acute renal failure and death in ICUs. ${ }^{5}$

We undertook this study to examine the predictive ability of RDW in comparison with other well-known disease severity scores among patients who were admitted to our ICU with AKI.

The primary objective is to determine which clinical prognostic score has the highest predictive values in patients with AKI requiring renal replacement therapy (RRT) using receiver-operating characteristic (ROC) curves. The secondary objective is to look for
1,2Department of Anaesthesiology and Critical Care, Sri Venkateswara
Institute of Medical Sciences, Tirupati, Andhra Pradesh, India
${ }^{3}$ Department of Nephrology, Sri Venkateswara Institute of Medical Sciences, Tirupati, Andhra Pradesh, India

Corresponding Author: Aloka Samantaray, Department of Anaesthesiology and Critical Care, Sri Venkateswara Institute of Medical Sciences, Tirupati, Andhra Pradesh, India, Phone: +91 9493547653, e-mail: aloksvims@gmail.com

How to cite this article: Nanjarapalle S, Samantaray A, Vishnubhotla S. Red Cell Distribution Width as a Severity Marker on the Outcome of Patients with Acute Kidney Injury on Renal Replacement Therapy. Indian J Crit Care Med 2020;24(2):95-98.

Source of support: Nil

Conflict of interest: None

the association between RDW and other prognostic predictive mortality models.

\section{Materials and Methods}

The study was approved by Institutional Ethics Committee, and a valid written informed consent or consent from legally acceptable relative was obtained from all the study participants. It was a prospective, observational single-centered, cohort study carried out in the ICU of a university teaching hospital over a period of 3 months.

All the adult patients of 18 years age or older and diagnosed with AKI or who develops AKI during ICU course of treatment and 
who need RRT constituted the study cohort. We have defined AKI according to Acute Kidney Injury Network (AKIN) criteria based on serum creatinine and urine output. ${ }^{6}$

We excluded patients unwilling to participate in the study, pregnant females, had history of packed RBC transfusion in the previous week, known hematological disorders (leukemia, myelodysplastic syndrome, and neoplastic metastases to bone marrow), recent chemotherapy, immunosuppression for solid organ transplantation, postsplenectomy, use of drugs known to induce changes in the morphology, and rheology of RBC (erythropoietin, pentoxifylline, cyclosporine, nitrates, etc.).

The following data were collected for each patient: age; gender; date and time of admission; complete physical and general examination; provisional diagnosis; comorbid conditions such as diabetes mellitus, hypertension, coronary artery disease, cerebrovascular accident, chronic obstructive pulmonary disease, chronic kidney disease, and chronic liver disease; and clinical and laboratory data were collected/noted, and necessary cultures were sent. Sequential organ failure assessment (SOFA) score, acute tubular necrosis-individual severity index (ATN-ISI), version II of acute physiology and chronic health evaluation (APACHE II), and version II of simplified acute physiology score (SAPS II) as a measure of severity of illness were calculated using the values of clinical and laboratory parameters at the time of admission. We also noted need for oxygen supplementation, duration of mechanical ventilation, ICU length of stay, and mortality. Doses of vasoactive medications were recorded hourly for the entire ICU stay, and the inotrope score (IS) and the vasoactive-inotropic score (VIS) were calculated as described previously. ${ }^{7}$ We calculated the mean IS and VIS after admission to the ICU to account for vasoactive support over time. The daily mean IS/VIS was calculated by summing the hourly doses every day and dividing by 24 . The final mean IS/VIS score for analysis was based on the sum of daily mean IS/VIS score divided by the number of days in ICU. Any escalation of inotropic or vasopressor dose after meeting clinical end points like cardiac arrest was not considered for analysis.

For the blood tests, hemogram and RDW were determined from whole blood using Sysmex XE_5000 analyzer (Sysmex Canada, Inc., Canada, USA). The reference range for RDW was between $11.5 \%$ and $14.5 \%$ in our center. We also recorded total leukocyte counts, blood urea, and serum creatinine at admission and as required during the course of treatment.

Indication for initiating RRT and mode of RRT (continuous RRT/ intermittent hemodialysis (IHD)/sustained low efficiency dialysis (SLED)/peritoneal dialysis) were noted.

Outcome of each patient was classified as survival to discharge or death (nonsurvivor) within 30 days after admission. The 30-day mortality was defined as death occurred within 30 days after the first admission. Patients who were discharged before 30 days, telephonic follow-up was done to ascertain survival or death.

\section{Statistical Analysis}

The discrimination of each severity score that predicts the risk of hospital mortality (APACHE, SOFA, ATN-ISI, SAPS II, RDW, VIS) was determined and compared by ROC curves for 30-day mortality. Area under the ROC curve (AROC) of $>0.9$ indicates excellent discrimination, whereas an area less than 0.5 means zero discriminatory power. An area from 0.5 to 0.7 suggests a low predictive discrimination, and a value greater than 0.7 implies a satisfactory discrimination power.
Based on survival data, the entire study cohort was divided into two groups, and comparisons were performed between 30-day survivors and nonsurvivors. Between these groups, continuous and categorical variables were analyzed, and results were expressed as mean \pm standard deviation (SD), median [interquartile range $(\mathrm{IQR})]$, or as number of cases and percentages as appropriate. Student's $t$ test was used to analyze normally distributed continuous variables, while Mann-Whitney $U$ test was used for no normally distributed continuous variables. Categorical variables were compared by means of Chi-square test. A worst case scenario analysis is undertaken where all patients who left against medical advice are considered to have died. We used Pearson correlation coefficient test to show the relationship between quantitative variables especially RDW. A $p$ value of less than 0.05 was considered statistically significant. All statistical tests performed were twotailed. A statistical analysis was performed using SPSS 20.0 software (IBM, Inc., New York, USA).

\section{Results}

During the study period, 30 consecutive patients with AKI were enrolled for the study. There are 26 medical and 4 surgical cases admitted to our ICU with renal failure. The distributions of 26 medical cases are: poisoning $(n=5)$, respiratory $(n=3)$, urosepsis $(n=5)$, traumatic brain injury $(n=1)$, diabetic ketoacidosis $(n=1)$, gastrointestinal $(n=4)$, vascular $(n=1)$, leptospirosis $(n=1)$, breast abscess $(n=1)$, septic shock $(n=2)$, cardio-renal syndrome $(n=1)$, and hepatic-renal syndrome $(n=1)$. The median length of stay in the ICU was 5 days, and the median duration of RRT was 7.5 days. Demographic characteristics of the patients are noted in Table 1. The median age was 54.5 years, and $70 \%$ of study population were male. Red blood cell distribution width ranged from $13.0 \%$ to $16.0 \%$ with a median value of $14.9 \%$ (IQR: $14.1-15.9 \%)$. In total, $66 \%$ patients (20 patients) required mechanical ventilation. The most common mode of RRT was IHD performed in 46.60\% (14 patients), followed by SLED in $36.7 \%$ (11 patients), continuous RRT in 10\% (3 patients), and peritoneal dialysis in $6.70 \%$ (2 patients). The mean number of days on mechanical ventilation and need for multiple inotropes are also higher among nonsurvivors. The most common mode of RRT in survivors is SLED (8 of 16 patients) and among nonsurvivors is IHD (10 of 14 patients). The median RDW in nonsurvivors and survivors was $15.4 \%$ and $14.4 \%$, respectively $(p<0.001)$. The overall 30 -day mortality of 30 patients was $53 \%$ (Table 1 ).

Table 2 displays the difference between observed parameter in the two groups of survivors and nonsurvivors. The patients who died were older, had higher leukocyte count, lower serum creatinine, and number of organ system involvement $(>2)$ as baseline parameter. The severity scores (APACHE II, SOFA, SAPS II, and ATN-ISI) of nonsurvivors at the time of admission to the ICU were higher as compared with that of survivors. The mean VIS is significantly higher among nonsurvivors $(4.8 \pm 5.6$ vs $0.8 \pm 3.2$; $p=0.026$ ). The mean ATN-ISI score in patients who died of AKI is $0.7 \pm 0.16$ and who survived is $0.5 \pm 0.14$.

The discrimination of APACHE, SOFA, ATN-ISI, SAPS II, and RDW is satisfactory with area $>0.7$, but RDW has the highest discriminatory power [AROC: $0.904,95 \%$ confidence interval $(\mathrm{Cl})$ : $0.791-1.000$ ]. The VIS has the least discriminatory power (AROC: 0.487, 95\% Cl: 0.257-0.716; Table 3).

Correlations of different disease severity parameters with RDW are presented in Table 4. Red blood cell distribution width has a statistically significant and strong correlation with SOFA score and 


\begin{tabular}{|c|c|c|c|}
\hline Patient variable & $\begin{array}{l}\text { Nonsurvivor } \\
(n=14)\end{array}$ & $\begin{array}{l}\text { Survivor } \\
(n=16)\end{array}$ & $p$ values \\
\hline Age in years & $47.5(18.1)$ & $52.4(16.7)$ & 0.454 \\
\hline Gender (male/female), $n$ & $12 / 4$ & $9 / 5$ & 0.694 \\
\hline Type of RRT, $n$ & & & 0.070 \\
\hline Continuous RRT & 2 & 1 & \\
\hline $\mathrm{HD}$ & 4 & 10 & \\
\hline PD & 3 & 0 & \\
\hline SLED & 8 & 3 & \\
\hline Duration of RRT & $7.4(4.4)$ & $7.5(4.2)$ & 0.934 \\
\hline Inotrope/vasopressor use, $n$ & & & 0.001 \\
\hline None & 1 & 10 & \\
\hline Single & 6 & 3 & \\
\hline$>1$ & 9 & 1 & \\
\hline $\begin{array}{l}\text { Mechanical ventilation (yes/ } \\
\text { no), } n\end{array}$ & $15 / 1$ & $5 / 9$ & 0.001 \\
\hline $\begin{array}{l}\text { Days of mechanical } \\
\text { ventilation, } n\end{array}$ & $7.8(5.6)$ & $3(4.3)$ & 0.014 \\
\hline Comorbidities & 9 & 6 & 0.051 \\
\hline None & 5 & 1 & \\
\hline Single & 2 & 7 & \\
\hline \multicolumn{4}{|l|}{$>1$} \\
\hline Serum creatinine (mg\%) & $6.2(2.9)$ & $5.1(3.1)$ & 0.333 \\
\hline Total leukocyte count $\left(\mathrm{mm}^{3}\right)$ & $\begin{array}{l}20,818 \\
(10,003)\end{array}$ & $\begin{array}{l}14,428 \\
(7,331)\end{array}$ & 0.055 \\
\hline ICU length of stay & $8.8(5.2)$ & $11.4(5.9)$ & 0.211 \\
\hline
\end{tabular}

Data are presented as mean (SD); $n$, number of patients; HD, hemodialysis; $\mathrm{PD}$, peritoneal dialysis

Table 2: Distribution of severity score between survivor and nonsurvivor

\begin{tabular}{lccl}
\hline Patient variable & $\begin{array}{l}\text { Nonsurvivor } \\
(n=14)\end{array}$ & $\begin{array}{l}\text { Survivor } \\
(n=16)\end{array}$ & $p$ values \\
\hline APACHE II & $19(5.5)$ & $11.4(4.7)$ & 0.000 \\
SOFA & $13.8(33)$ & $8.5(3.3)$ & 0.000 \\
ATN-ISI & $0.7(0.16)$ & $0.5(0.14)$ & 0.018 \\
SAPS II & $53.1(10.9)$ & $36(10.8)$ & 0.066 \\
RDW (\%) & $15.58(0.6)$ & $14.26(0.6)$ & 0.000 \\
VIS & $4.8(5.6)$ & $0.8(3.2)$ & 0.026 \\
\hline
\end{tabular}

Data are presented as mean (SD)

APACHE II $(p=0.000, r=0.603)$. Both SAPS $(p=0.013, r=0.447)$ and ATN-ISI score $(p=0.006, r=0.493)$ bear a statistically significant but moderate correlation with RDW (Table 4).

\section{Discussion}

Our study finding demonstrated that high RDW has a higher discriminatory power to predict poor clinical outcome in patients who needed RRT in the ICUs and also has a good correlation with other disease severity scores (SOFA and APACHE II). Multiple factors have been identified as a predictor for poor outcome of critically ill patients undergoing hemodialysis. ${ }^{8-10}$

In addition to being linked to renal function, an elevated RDW has also been associated with higher mortality in patients with AKI who are receiving continuous RRT.11
A Lithuanian study ${ }^{12}$ opined that lethal patient outcome is related to Glasgow coma score, mean arterial blood pressure, preoperative serum creatinine, and postoperative platelet count in patients with AKI requiring RRT after cardiac surgery, whereas APACHE II score and ATN-ISI score have a lower predictive value with AROC lying between 0.5 and 0.7 . In contrast, our study result also demonstrates a satisfactory AROC for both APACHE II (AROC: 0.828) and ATN-ISI (AROC: 0.743). This difference could be because, unlike the Lithuanian study, our study was conducted in medical ICU with higher APACHE admission score and sepsis contribute to the cause of AKI in large number of patients. Similar to Lithuanian study, two more studies from Russia ${ }^{13}$ and Brazil ${ }^{14}$ also used APACHE II, SOFA, SAPS II, and ATN-ISI as prognostic models that predict the risk of hospital mortality. The value of the AROC of the three scales was obtained in a close range of 0.821 (APACHE II) to 0.855 (SOFA), 0.842 (ATN-ISI) which is a very good indicator. Our study also demonstrated a very discriminatory power for the same prognostic variables with AROC lying in the range of $0.742-0.828$.

A study from a large referral hospital from North India showed a graded relationship between RDW and 30-day mortality but failed to prove increased RDW as an independent predictor of 30-day mortality. ${ }^{15}$ However, in their study 146 patients from a cohort of 200 patients admitted with sepsis had renal failure, and they demonstrated an significantly increased RDW in patients associated with renal failure $(p=0.031)$. Their result further revealed that unlike renal failure other organ failure is not associated with an increase in RDW. Two Chinese studies ${ }^{16,17}$ separately in patients after noncardiac surgery and in patients with ST-elevation myocardial infarction had demonstrated that increased RDW is associated with a higher mortality rate but both the studies also opined that discrimination power of RDW is limited with small AROCs (0.562-0.614) and so is a marker with a very low prognostic accuracy. However, another Chinese study ${ }^{18}$ which is a prospective observational study done in adult coronary care unit (CCU) concluded that RDW is not only an independent predictor of AKI but also a good predictor for mortality (AROC: $0.632 ; 95 \% \mathrm{Cl}$ : 0.556-0.709) in patients in the CCU. A similar conclusion was reached in our study where we demonstrated that RDW has a better discriminatory power for mortality and also had good relation with APACHE II score and SOFA score (Table 4).

The mechanism for the association between RDW values and mortality is not fully understood. Several plausible explanations have been suggested in prior reports. Systemic inflammation has been shown to predict progressive illness, cardiovascular death, and mortality in ICU patients. ${ }^{15}$ A recent study demonstrated that RDW is related to C-reactive protein (CRP) levels, which is an acute phase reactant and is significantly associated with worst outcomes.

The VIS was developed to quantify vasoactive and inotropic support after cardiac surgery in pediatric patients but may be useful in adults as well. An American study ${ }^{19}$ applied VIS score in adult population and demonstrated that temporary biventricular pacing lower the perioperative requirements for vasoactive medication support, as measured by the VIS. Our study shows that mean VIS is significantly higher among nonsurvivors ( $4.8 \pm 5.6$ vs $0.8 \pm 3.2 ; p=$ $0.026)$. However, the predictive ability of VIS is very low in our study (AROC: 0.487 ). This could be because only 10 of 30 patients needed more than two vasopressor/inotropes, and second, the initiation and escalation of the vasopressor/inotropes was at the discretion of the treating physician, and a standard protocol was not followed.

There were several limitations to our study, including single-center data in medical ICU setting and small sample size. 
RDW as a Severity Marker on the Outcome of Patients with AKI on Renal Replacement Therapy

Table 3: Discriminatory power of six disease severity scores

\begin{tabular}{lllllll}
\hline & APACHE II & SOFA & ATN-ISI & SAPS II & RDW & VIS \\
\hline AROC & 0.828 & 0.850 & 0.743 & 0.857 & 0.904 & 0.487 \\
SE & 0.076 & 0.068 & 0.09 & 0.071 & 0.058 & 0.071 \\
$95 \% \mathrm{Cl}$ & $0.079-0.977$ & $0.716-0.984$ & $0.567-0.920$ & $0.718-0.996$ & $0.791-1.000$ & $0.257-0.716$ \\
\hline
\end{tabular}

$\mathrm{SE}$, standard error

Table 4: Correlation of red blood cell distribution width and other prognostic disease severity scores

\begin{tabular}{lll}
\hline Variable & $R^{2}$ (Pearson correlation) & $p$ \\
\hline APACHE II & 0.637 & 0.000 \\
SOFA & 0.650 & 0.000 \\
ATN-ISI & 0.506 & 0.002 \\
SAPS II & 0.406 & 0.005 \\
VIS & 0.075 & 0.346 \\
\hline
\end{tabular}

So, the study results cannot be extrapolated to critical ill patients in surgical ICUs. Therefore, we suggest conducting future studies with larger sample size. The strength of this study is that it is the first report demonstrating the strong association between RDW and other disease severity scoring system in adult patients with AKI and necessitating dialysis.

To conclude, our study has added to the evidence showing that RDW has a better predictive ability for mortality and good correlation with APACHE II and SOFA disease severity scoring models.

\section{Author Contributions}

Sunil Nanjarapalle, Aloka Samantaray, Sivakumar Vishnubhotla contributed to concepts, design, definition of intellectual content, experimental studies, manuscript preparation, manuscript editing, manuscript review and were guarantors. Aloka Samantaray and Sivakumar Vishnubhotla performed literature search, data analysis and statistical analysis. Sunil Nanjarapalle performed clinical studies and data acquisition.

\section{References}

1. Kasuno K, Shirakawa K, Yoshida H, Mori K, Kimura H, Takahashi N, et al. Renal redox dysregulation in AKI: application for oxidative stress marker of AKI. Am J Physiol Renal Physiol 2014;307(12):F1342-F1351. DOI: 10.1152/ajprenal.00381.2013.

2. Feng Y, Liu Y, Wang L, Cai X, Wang D, Wu K, et al. Sustained oxidative stress causes late acute renal failure via duplex regulation on $p 38$ MAPK and Akt phosphorylation in severely burned rats. PLoS One 2013;8(1):e54593. DOI: 10.1371/journal.pone.0054593.

3. Lippi G, Targher G, Montagnana M, Salvagno GL, Zoppini G, Guidi $\mathrm{GC}$, et al. Relation between red blood cell distribution width and inflammatory biomarkers in a large cohort of unselected outpatients. Arch Pathol Lab Med 2009;133(4):628-632. DOI: 10.1043/1543-2165133.4.628.

4. Jo YH, Kim K, Lee JH, Kang C, Kim T, Park HM, et al. Red cell distribution width is a prognostic factor in severe sepsis and septic shock. Am J Emerg Med 2013;31(3):545-548. DOI: 10.1016/j.ajem.2012.10.017.

5. Bhadade R, De'Souza R, Harde MJ, Mehta KS, Bhargava P. A prospective study of acute kidney injury according to KDIGO definition and its mortality predictors. J Assoc Physicians India 2016;64(12):22-28.

6. Mehta RL, Kellum JA, Shah SV, Molitoris BA, Ronco C, Warnock DG, et al. Acute kidney injury network: report of an initiative to improve outcomes in acute kidney injury. Crit Care 2007;11(2):R31. DOI: 10.1186/ cc5713.

7. Gaies MG, Gurney JG, Yen AH, Napoli ML, Gajarski RJ, Ohye RG, et al. Vasoactive-inotropic score as a predictor of morbidity and mortality in infants after cardiopulmonary bypass. Pediatr Crit Care Med 2010;11(2):234-238. DOI: 10.1097/PCC.0b013e3181b806fc.

8. Mukhoedova TV, Lomivorotov VN, Kolesnikov SV, Litasova EE, Malov AA, Borisov AS. Prediction of the outcomes of renal replacement therapy for acute renal failure after cardiosurgical interventions. Anesteziol Reanimatol 2005;2:53-58.

9. Allegretti AS, Steele DJ, David-Kasdan JA, Bajwa E, Niles JL, Bhan I. Continuous renal replacement therapy outcomes in acute kidney injury and end-stage renal disease: a cohort study. Crit Care 2013;17(3):R109. DOI: 10.1186/cc12780.

10. Prasanth TS, Remya KA, Resmi NR, Philipose R, Vijayakumar K. Acute renal failure renal replacement therapy \& outcome. Urol Nephrol Open Access J 2015;2(5):00056. DOI: 10.15406/unoaj.2015.02. 00056.

11. Oh HJ, Park JT, Kim JK, Yoo DE, Kim SJ, Han SH, et al. Red blood cell distribution width is an independent predictor of mortality in acute kidney injury patients treated with continuous renal replacement therapy. Nephrol Dial Transplant 2012;27(2):589-594. DOI: 10.1093/ ndt/gfr307.

12. Skarupskienè I, Adukauskienè $D$, Kuzminskienè J, Rimkutè $L$, Balčiuvienè $\mathrm{V}$, Žiginskienè $\mathrm{E}$, et al. Mortality prediction in patients with acute kidney injury requiring renal replacement therapy after cardiac surgery. Medicina (Kaunas) 2017;53(4):217-223. DOI: 10.1016/ j.medici.2017.06.003.

13. Malov AA, Borisov AS, Lomivorotov VV, Efremov SM, Ponomarev DN, Mukhoedova TV, et al. Mortality prediction in patients with dialysis-dependent acute kidney injury after cardiac surgery with cardiopulmonary bypass. Heart Lung Circ 2014;23(4):325-331. DOI: 10.1016/j.hlc.2013.10.082.

14. Lima EQ, Dirce MT, Castro I, Yu L. Mortality risk factors and validation of severity scoring systems in critically ill patients with acute renal failure. Ren Fail 2005;27(5):547-556. DOI: 10.1080/08860220500198771.

15. Jandial A, Kumar S, Bhalla A, Sharma N, Varma N, Varma S. Elevated red cell distribution width as a prognostic marker in severe sepsis: a prospective observational study. Indian J Crit Care Med 2017;21(9):552-562. DOI: 10.4103/ijccm.IJCCM_208_17.

16. Cheung YN, Shum HP, Chan KC, Yan WW. Preoperative red cell distribution width: not a useful prognostic indicator for 30-day mortality in patients who undergo major- or ultra-major noncardiac surgery. Indian J Crit Care Med 2016;20(11):647-652. DOI: 10.4103/0972-5229.194008.

17. Sun XP, Chen WM, Sun ZJ, Ding XS, Gao XY, Liang SW, et al. Impact of red blood cell distribution width on long-term mortality in patients with ST-elevation myocardial infarction. Cardiology 2014;128(4): 343-348. DOI: 10.1159/000359994.

18. Hu Y, Liu H, Fu S, Wan J, Li X. Red blood cell distribution width is an independent predictor of AKI and mortality in patients in the coronary care unit. Kidney Blood Press Res 2017;42(6):1193-1204. DOI: 10.1159/000485866.

19. Nguyen HV, Havalad V, Aponte-Patel L, Murata AY, Wang DY, Rusanov $A$, et al. Temporary biventricular pacing decreases the vasoactiveinotropic score after cardiac surgery: a substudy of a randomized clinical trial. J Thorac Cardiovasc Surg 2013;146(2):296-301. DOI: 10.1016/j.jtcvs.2012.07.020. 